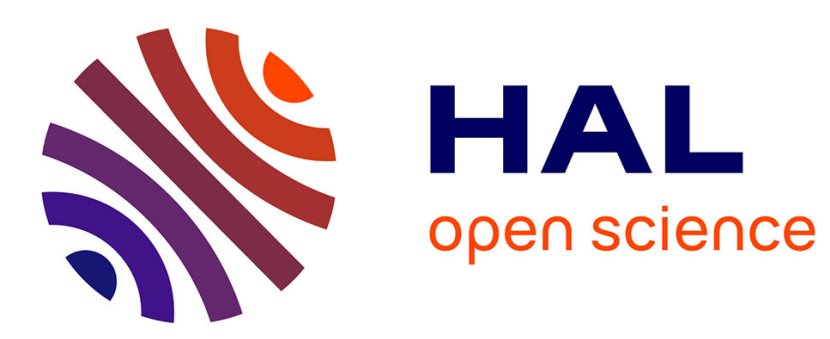

\title{
A mathematical study of quantum revivals and quantum fidelity
}

\author{
M. Combescure
}

\section{To cite this version:}

M. Combescure. A mathematical study of quantum revivals and quantum fidelity. Bedlewo Conference on Operators Theory and Applications, Jul 2004, Bedlewo, Poland. pp.15. in2p3-00022208

\section{HAL Id: in2p3-00022208 https://hal.in2p3.fr/in2p3-00022208}

Submitted on 6 Sep 2004

HAL is a multi-disciplinary open access archive for the deposit and dissemination of scientific research documents, whether they are published or not. The documents may come from teaching and research institutions in France or abroad, or from public or private research centers.
L'archive ouverte pluridisciplinaire HAL, est destinée au dépôt et à la diffusion de documents scientifiques de niveau recherche, publiés ou non, émanant des établissements d'enseignement et de recherche français ou étrangers, des laboratoires publics ou privés. 


\title{
A mathematical study of Quantum Revivals and Quantum Fidelity
}

\author{
Monique Combescure \\ Laboratoire de Physique Théorique, CNRS - UMR 8627 \\ Université de Paris XI, Bâtiment 210, F-91405 ORSAY Cedex, France \\ and \\ IPNL, Bâtiment Paul Dirac \\ 4 rue Enrico Fermi, Université Lyon-1 \\ F.69622 VILLEURBANNE Cedex, France \\ email monique.combescure@ipnl.in2p3.fr
}

3 septembre 2004

\begin{abstract}
Résumé
In this paper we present some results obtained recently, partly in collaboration with Didier Robert, about "Quantum Revivals" and "Quantum Fidelity", mainly in the semiclassical framework. We also descibe the exact properties of the Quantum Fidelity (also called Loschmidt Echo) for the case of explicit quadratic plus inverse quadratic time-periodic Hamiltonians and establish that the quantum fidelity equals one for exactly the times where the classical fidelity is maximal.
\end{abstract}

Proceedings of the Bedlewo Conference "Operator Theory and Applications in Mathematical Physics" (July 2004) 


\section{Introduction}

The quantum return probability is the modulus of the overlap between an initial wavepacket and its time evolution governed by Schrödinger equation. When this quantity happens to equal one for some time $t$, then the system is said to exhibit "Quantum Revivals".

$$
R(t):=|\langle\psi, U(t, 0) \psi\rangle|
$$

When the Hamiltonian $\hat{H}(t)$ (possibly time-dependent) is assumed to be perturbed $\hat{H}_{g}(t):=\hat{H}(t)+g V$, then we can compare the evolutions generated by $\hat{H}(t)$ and $\hat{H}_{g}(t)$ respectively, acting on the same initial state $\psi$ :

$$
F(t):=\left\langle U(t, 0) \psi, U_{g}(t, 0) \psi\right\rangle
$$

which is the measure of the "Quantum Fidelity" in the state $\psi$ along the evolution. Of course $F(0)=1$ and $F(t) \equiv 1$ if $g=0$.

Thus the decrease in $t$ of $F(t)$ measures the lack of fidelity due to the perturbation.

Both Quantum Revivals and Quantum Fidelity have attracted much recent interest in the physics literature (see references). Notably, it has been heuristically claimed that the decrease in time of the Quantum Fidelity allows to distinguish between systems having regular versus chaotic classical evolution.

In our study, we consider the semiclassical regime for both quantities, using coherent states as initial wavepackets $\psi$.

We also perform exact calculations of the Quantum Fidelity in the case of the singular time-periodic Harmonic Oscillator, with initial wavepackets $\psi$ being "generalized coherent states" in the sense of Perelomov, showing that they do not decrease to zero as time evolves, but present recurrences to 1 exactly at the values of times where the classical fidelity is maximal. More specifically we consider Hamiltonians :

$$
\hat{H}(t):=\frac{P^{2}}{2}+f(t) \frac{Q^{2}}{2}
$$

and

$$
\hat{H}_{g}(t):=\hat{H}(t)+\frac{g^{2}}{Q^{2}}
$$

where the real constant $g$ is the size of the perturbation, and $f$ is a T-periodic function of time, and we perform an exact calculation of :

$$
F(t):=\left\langle U(t, 0) \psi, U_{g}(t) \psi\right\rangle
$$

where $U(t, 0)$ (resp $\left.U_{g}(t, 0)\right)$ is the quantum evolution generated by $\hat{H}(t)$, (resp. $\left.\hat{H}_{g}(t)\right)$. 


\section{Semiclassical Quantum Revivals}

We use the "Coherent States" of the Harmonic Oscillator : let $\varphi_{0}$ be the ground state of the Harmonic Oscillator $\hat{P}^{2} / 2+\hat{Q}^{2} / 2$ in dimension $n$, where $\hat{Q}$ (resp. $\hat{P}$ ) is the operator of multiplication by $x$ (resp. the derivation operator $\hat{P}:=-i \hbar \nabla)$ in the Hilbert space of quantum states $\mathcal{H}=L^{2}\left(\mathbb{R}^{n}\right)$.

The Weyl-Heisenberg unitary translation operator by $\alpha:=(q, p)$ is defined as :

$$
\hat{T}(\alpha):=\exp \left(i \frac{p \cdot \hat{Q}-q \cdot \hat{P}}{\hbar}\right)
$$

and the coherent state $\varphi_{\alpha}$ is defined as follows :

$$
\varphi_{\alpha}:=\hat{T}(\alpha) \varphi_{0}
$$

Now consider a classical trajectory in phase space induced by the Hamiltonian $H:=$ $\frac{p^{2}}{2}+V(q):$

$$
\alpha \mapsto \alpha_{t}:=\left(q_{t}, p_{t}\right)
$$

Let $S(t)$ be the classical action along this trajectory, and $M_{t}$ be the Hessian matrix of $H$ taken at point $\alpha_{t}$ :

$$
\left(M_{t}\right)_{i, j}:=\frac{\partial^{2} H}{\partial \alpha_{i} \partial \alpha_{j}}\left(q_{t}, p_{t}\right)
$$

It is a real symmetric $2 n \times 2 n$ matrix. The linearized flow (or Stability Matrix) is obtained by solving the differential equation :

$$
\dot{F}_{t}=J M_{t} F_{t}
$$

where $J$ is the symplectic matrix :

$$
J=\left(\begin{array}{cc}
0 & \mathbb{1} \\
-\mathbb{1} & 0
\end{array}\right)
$$

with initial data

$$
F_{0}=\mathbb{1}
$$

Clearly $F_{t}$ is a symplectic matrix, ie satisfies for any $t$

$$
\tilde{F}_{t} J F_{t}=J
$$

Namely

$$
\frac{d}{d t}\left(\tilde{F}_{t} J F_{t}\right)=-\tilde{F}_{t} M_{t} J J F_{t}+\tilde{F}_{t} J J M_{t} F_{t}=\tilde{F}_{t} M_{t} F_{t}-\tilde{F}_{t} M_{t} F_{t}=0
$$

and therefore $\tilde{F}_{t} J F_{t}$ is a constant $2 n \times 2 n$ matrix equal to $J$ since $F_{0}=\mathbb{1}$. 
To this symplectic matrix can be associated a unitary operator $\hat{R}(F)$ in the Hilbert space $\mathcal{H}$, acting as expected :

$$
\hat{R}(F)^{-1}\left(\begin{array}{c}
\hat{Q} \\
\hat{P}
\end{array}\right) \hat{R}(F)=F\left(\begin{array}{c}
\hat{Q} \\
\hat{P}
\end{array}\right)
$$

Now we are in a position to give the semiclassical approximation for the quantum evolution of coherent states (see [8])

Proposition 2.1 Under suitable assumptions on $V$, there exists a constant $\epsilon(\hbar, t)$, small as $\hbar$ goes to zero, such that if $U(t, 0)$ is the quantum evolution operator associated to the Weyl quantization of $H$ one has :

$$
\left\|U(t, 0) \varphi_{\alpha}-e^{i \delta_{t} / \hbar} \hat{T}\left(\alpha_{t}\right) \hat{R}\left(F_{t}\right) \varphi_{0}\right\| \leq \epsilon(\hbar, t)
$$

where $\delta_{t}:=S(t)-\left(p_{t} \cdot q_{t}-p . q\right) / 2$

Thus, up to a controlable error, the recurrence probability $\left|\left\langle\varphi_{\alpha}, U(t, 0) \varphi_{\alpha}\right\rangle\right|$ can be replaced with

$$
\tilde{R}(t):=\left|\left\langle\hat{T}(\alpha) \varphi_{0}, \hat{T}\left(\alpha_{t}\right) \hat{R}\left(F_{t}\right) \varphi_{0}\right\rangle\right|=\left|\left\langle\hat{T}\left(\alpha-\alpha_{t}\right) \varphi_{0}, \hat{R}\left(F_{t}\right) \varphi_{0}\right\rangle\right|
$$

We shall now make use of a beautiful result by Mehlig and Wilkinson [22], that gives the Weyl covariant symbol of the metaplectic operators. For a complete mathematical proof see our paper [9].

Proposition 2.2 Let $F$ be a symplectic $2 n \times 2 n$ matrix not having 1 as eigenvalue. Then the associated metaplectic operator $\hat{R}(F)$ can be written as

$$
\hat{R}(F)=\frac{h^{-n} \gamma_{F}}{|\operatorname{det}(\mathbb{1}-F)|^{1 / 2}} \int_{\mathbb{R}^{2 n}} d z \hat{T}(z) e^{i z . A z / 2 \hbar}
$$

where $\gamma_{F}$ is a complex number of modulus 1, and

$$
A:=\frac{J}{2}(F+\mathbb{1})(F-\mathbb{1})^{-1}
$$

We now set

$$
z_{t}:=\alpha-\alpha_{t}
$$

Then using Proposition 2.2, we have :

$$
\tilde{R}(t)=h^{-n}\left|\operatorname{det}\left(\mathbb{1}-F_{t}\right)\right|^{-1 / 2}\left|\int d z\left\langle\hat{T}\left(z_{t}\right) \varphi_{0}, \hat{T}(z) \varphi_{0}\right\rangle e^{i z . A z / 2 \hbar}\right|
$$




$$
\begin{gathered}
=h^{-n}\left|\operatorname{det}\left(\mathbb{1}-F_{t}\right)\right|^{-1 / 2}\left|\int d z \exp \left(\frac{i z \cdot A z}{2 \hbar}+\frac{i z \cdot J z_{t}}{2 \hbar}-\frac{1}{4 \hbar}\left(z-z_{t}\right)^{2}\right)\right| \\
=h^{-n}\left|\operatorname{det}\left(\mathbb{1}-F_{t}\right)\right|^{-1 / 2} e^{-z_{t}^{2} / 4 \hbar}\left|\int d z \exp \left(-\frac{1}{2 \hbar} z \cdot\left(\frac{1}{2}-i A\right) z+\frac{1}{2 \hbar} z \cdot(J+i \mathbb{1}) z_{t}\right)\right|
\end{gathered}
$$

Now by using the calculus of Fourier Transforms of Gaussians, we get :

$$
\tilde{R}(t)=\left|\operatorname{det}\left(\mathbb{1}-F_{t}\right)\left(\frac{\mathbb{1}}{2}-i A\right)\right|^{-1 / 2}\left|\exp \left(-\frac{z_{t}^{2}}{4 \hbar}-\frac{1}{4 \hbar} z_{t} \cdot K z_{t}\right)\right|
$$

where the matrix $K$ is given by

$$
K:=(J-i \mathbb{1})(\mathbb{1}-2 i A)^{-1}(J+i \mathbb{1})
$$

Now we have the following remarkable result :

$$
\frac{\mathbb{1}}{2}-i A=N(F-\mathbb{1})^{-1}
$$

where

$$
N:=\frac{1}{2}(F(\mathbb{1}-i J)-(\mathbb{1}+i J))
$$

so that

$$
\left|\operatorname{det}(\mathbb{1}-F)\left(\frac{\mathbb{1}}{2}-i A\right)\right|^{-1 / 2}=|\operatorname{det} N|^{-1 / 2}
$$

But $N$ has the important following property

\section{Lemma 2.3}

$$
|\operatorname{det} N| \geq 1
$$

and

$|\operatorname{det} N|=1 \quad \Longleftrightarrow F$ is unitary

This result has been established in full generality in [9], but we shall here indicate the calculus in dimension $n=1$. The symplectic matrix $F$ has now the simple form

$$
F=\left(\begin{array}{ll}
a & b \\
c & d
\end{array}\right)
$$

with $a d-b c=1$ to ensure the symplecticity. Using the form given above for $N$ we easily get

$$
\operatorname{det} N=-\frac{1}{2}(a+d+i(b-c))
$$

so that

$$
|\operatorname{det} N|^{2}=\frac{1}{4}\left((a+d)^{2}+(b-c)^{2}\right)=\frac{1}{4}\left(4+(a-d)^{2}+(b+c)^{2}\right) \geq 1
$$

with equality to $1 \Longleftrightarrow \quad a=d, b=-c$, in which case $F$ is just unitary (rotation).

Thus we get the following result 
Theorem 2.4 Denoting by $z_{t}$ the following distance $z_{t}:=\alpha-\alpha_{t}$, the complete return probability has the following semiclassical estimate:

$$
\left.|R(t)-| \operatorname{det} N\right|^{-1 / 2}\left|\exp \left(-\frac{z_{t}^{2}}{4 \hbar}-\frac{1}{4 \hbar} z_{t} \cdot K z_{t}\right)\right| \mid \leq \epsilon(\hbar, t)
$$

Thus if $\alpha$ lies on a classical periodic orbit $\gamma$ with period $T_{\gamma}$, the exponential is just 1 ; furthermore the prefactor is $1 \Longleftrightarrow F_{T_{\gamma}}$ is unitary, in which case we have almost semiclassical recurrence.

$$
R\left(T_{\gamma}\right) \geq 1-\epsilon(\hbar, t)
$$

Note that $\epsilon\left(\hbar, T_{\gamma}\right)=O\left(\hbar^{\varepsilon}\right)$ provided $T_{\gamma} \leq \lambda|\log \hbar|$ for some $\lambda$ given by the classical dynamics.

\section{$3 \quad$ The Quantum Fidelity}

Let $\hat{H}$ be a quantum Hamiltonian, and $\hat{H}_{g}:=\hat{H}+g V$ be a perturbation of it $(g$ small). The quantum fidelity at time $t$ in the state $\psi$ is given as

$$
F(t):=\left|\left\langle e^{-i t \hat{H} / \hbar} \psi, e^{-i t \hat{H}_{g} / \hbar} \psi\right\rangle\right|
$$

Remark 3.1 If $\psi=\psi_{j}$ is an eigenstate of $\hat{H}$ (resp. $\hat{H}_{g}$ ), then the fidelity is nothing else that the "return probability" $\left|\left\langle\psi, e^{-i t \hat{H}_{g} / \hbar} \psi\right\rangle\right|\left(\right.$ resp. $\left.\left|\left\langle\psi, e^{-i t \hat{H} / \hbar} \psi\right\rangle\right|\right)$

Remark 3.2 Clearly $F(0)=1$, and $F(t) \equiv 1$ if $g=0$. One expects that if $g \neq 0$ then $F(t)$ will decrease as time increases. Furthermore it is believed that this decrease could be significantly different for an associated classical dynamics being regular versus chaotic. No exact result has been established up to now.

One can semiclassically estimate this quantum fidelity along the same lines as the return probability above.

Theorem 3.3 Let $\alpha_{t}$ (resp. $\left.\alpha^{\prime}(t)\right)$ be the classical phase-space point reached by the trajectory governed by Hamiltonian $H$ (resp. $H_{g}$ ), starting from the same point $\alpha$ at time 0. Then the following estimate holds true:

$$
\left|F(t)-C_{t} \exp \left\{-\frac{1}{4 \hbar}\left(F_{t}^{\prime}\left(\alpha_{t}^{\prime}-\alpha_{t}\right)\right)^{2} K(\alpha, g, t)\right\}\right| \leq \hbar^{1 / 2} L(\alpha, g, t)
$$

where $F_{t}^{\prime}$ is the stability matrix for the dynamics generated by $H_{g}$, and $C_{t}, K(\alpha, g, t), L(\alpha, g, t)$ are positive controlable constants.

Remark 3.4 The proof of this statement is contained in our paper [9]. The important fact to notice is that the classical infidelity $\alpha_{t}-\alpha_{t}^{\prime}$ is an important quantity to estimate in $t$ and in $g$. 
We now come to an interesting particular case where the quantum fidelity can be computed exactly. The Hamiltonians considered are time-periodic and have the following form :

$$
\begin{aligned}
H_{0}(t) & :=\frac{p^{2}}{2}+f(t) \frac{x^{2}}{2} \\
H_{g}(t) & :=H_{0}(t)+\frac{g^{2}}{x^{2}}
\end{aligned}
$$

where $t \mapsto f$ is a T-periodic function, and $g$ a real constant.

We denote by $\hat{H}_{0}(t)$ and $\hat{H}_{g}(t)$ the corresponding selfadjoint operators in $\mathcal{H}=L^{2}(\mathbb{R})$. The initial states we shall consider are generalized coherent states in the sense of Perelomov ([23]) adapted to the underlying algebra SU(1,1). Let

$$
\begin{gathered}
K_{0}:=\frac{\hat{Q}^{2}+\hat{P}^{2}}{4}+\frac{g^{2}}{2 \hat{Q}^{2}}=2 \hat{H}_{g} \\
K_{ \pm}=\frac{\hat{P}^{2}-\hat{Q}^{2}}{4} \mp i \frac{\hat{Q} \cdot \hat{P}+\hat{P} \cdot \hat{Q}}{4}-\frac{g^{2}}{2 \hat{Q}^{2}}
\end{gathered}
$$

They satisfy :

$$
\begin{gathered}
{\left[K_{0}, K_{ \pm}\right]= \pm K_{ \pm}} \\
{\left[K_{-}, K_{+}\right]=2 K_{0}} \\
K_{-}=K_{+}^{*}
\end{gathered}
$$

Let $\psi$ be the ground state of $\hat{H}_{g}$ :

$$
\hat{H}_{g} \psi=\left(\alpha+\frac{1}{2}\right) \psi
$$

with $\alpha=\frac{1}{2}+\sqrt{\frac{1}{4}+2 g^{2}}$. It also annihilates $K_{-}$:

$$
K_{-} \psi=0
$$

and has the following form :

$$
\psi(x)=c x^{\alpha} e^{-x^{2} / 2}
$$

where $\mathrm{c}$ is a normalization constant such that $\|\psi\|=1$.

For $\beta \in \mathbb{C}$, we define the unitary operator

$$
\hat{S}(\beta):=\exp \left(\beta K_{+}-\bar{\beta} K_{-}\right)
$$

and the generalized coherent states as

$$
\psi_{\beta}:=\hat{S}(\beta) \psi
$$


Let $U_{0}(t, 0)$ and $U_{g}(t, 0)$ be the quantum unitary evolution operators generated by $\hat{H}_{0}(t)$ and $\hat{H}_{g}(t)$. We shall study the quantum fidelity (without absolute value) :

$$
F(t):=\left\langle U_{0}(t, 0) \psi_{\beta}, U_{g}(t, 0) \psi_{\beta}\right\rangle
$$

We shall first study the particular case $g=1$ (whence $\alpha=2$ ). Then $\psi$ is obviously a simple linear combination of the eigenstates $\varphi_{0}$ and $\varphi_{2}$ of the harmonic oscillator.

We have the following explicit result (see [7]) :

Proposition 3.5 Let $z(t)$ be a complex solution of the linear differential equation (Hill's equation) :

$$
\ddot{z}+f z=0
$$

We define its polar decomposition by

$$
z(t)=e^{u+i \theta}
$$

where $t \mapsto u$ and $t \mapsto \theta$ are real, and consider the following initial data:

$$
\begin{gathered}
u(0)=u_{0} \\
\dot{u}(0)=\dot{u}_{0} \\
\theta(0)=\theta_{0} \\
\dot{\theta}(0)=e^{-2\left(u_{0}-\epsilon\right)}
\end{gathered}
$$

Let $\hat{H}_{g}=\frac{\hat{P}^{2}+\hat{Q}^{2}}{2}+\frac{g^{2}}{\hat{Q}^{2}}$. Then we have :

$$
U_{g}(t, 0)=e^{i \dot{u} \hat{Q}^{2} / 2} e^{-i(u-\epsilon)(\hat{Q} \cdot \hat{P}+\hat{P} \cdot \hat{Q}) / 2} e^{-i\left(\theta-\theta_{0}\right) \hat{H}_{g}} e^{i\left(u_{0}-\epsilon\right)(\hat{Q} \cdot \hat{P}+\hat{P} \cdot \hat{Q}) / 2} e^{-i \dot{u}_{0} \hat{Q}^{2} / 2}
$$

The same formula holds true for $U_{0}(t, 0)$ with $\hat{H}_{g}(t)$ replaced by $\hat{H}_{0}(t)$.

An important fact to notice is that the constants $u_{0}, \dot{u}_{0}, \theta_{0}, \epsilon$ can be adjusted, given any $\beta \in \mathbb{C}$ such that

$$
\hat{S}(\beta) \psi=e^{i \dot{u}_{0} \hat{Q}^{2} / 2} e^{-i\left(u_{0}-\epsilon\right)(\hat{Q} \cdot \hat{P}+\hat{P} \cdot \hat{Q}) / 2} e^{-i \theta_{0} \hat{H}_{g}} \psi
$$

Then $U_{g}(t, 0) \psi$ and $U_{0}(t, 0) \psi$ have simple explicit form (see [7]), leading to a very simple form of the fidelity :

Theorem 3.6 Let $g=1$. Then the fidelity is just given by

$$
F(t)=\frac{2}{3}+\frac{1}{3} e^{-2 i(\theta(t)-\theta(0))}
$$


Let us remark that $\theta(t)$ is just given by the formula

$$
\theta(t)-\theta(0)=e^{2 \epsilon} \int_{0}^{t} e^{-2 u(s)} d s
$$

\section{Study of the Hill's equation}

$$
\ddot{z}+f z=0
$$

\section{-Stable case :}

$u$ is T-periodic, and thus $\theta(t)$ grows from $-\infty$ to $+\infty$ when $t$ varies from $-\infty$ to $+\infty$. Therfore there exists an infinite sequence $\left(t_{k}\right)_{k \in \mathbb{Z}}$ such that

$$
\theta\left(t_{k}\right)-\theta(0)=2 k \pi
$$

in which case $F\left(t_{k}\right)=1$, ie the quantum fidelity is perfect. Moreover there exists an infinite sequence $\left(t_{k}^{\prime}\right)_{k \in \mathbb{Z}}$ such that

$$
\theta\left(t_{k}^{\prime}\right)-\theta(0)=(2 k+1) \pi
$$

in which case $F\left(t_{k}^{\prime}\right)=1 / 3$. We thus have the following picture :

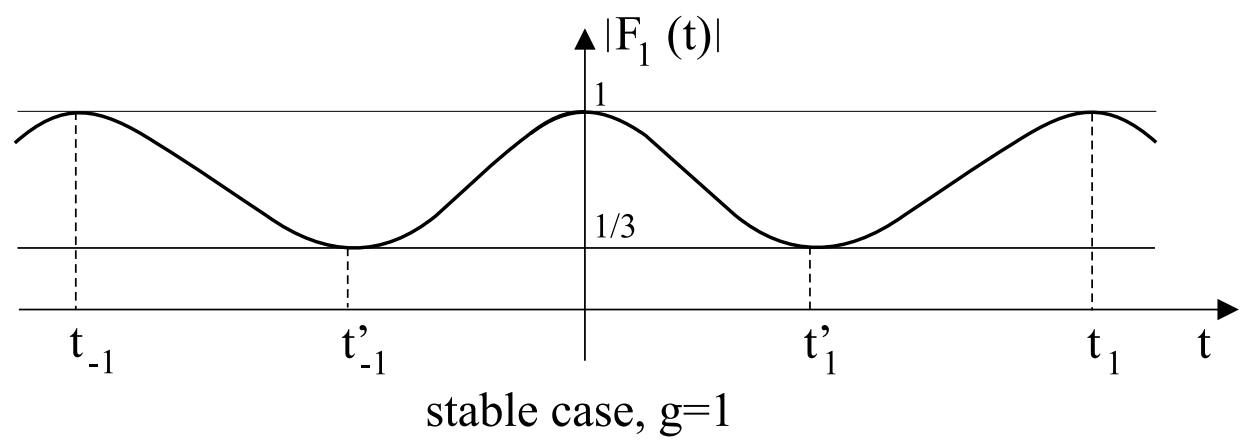

\section{- unstable case :}

$u(t) \simeq \pm \lambda t$ with, say, $\lambda>0$ (Lyapunov exponent) in which case $\theta(t) \rightarrow$ cst as $t \rightarrow+\infty$ and $\theta(t) \rightarrow-\infty$ exponentially as $t \rightarrow-\infty$ (or vice-versa). We thus have the following picture :

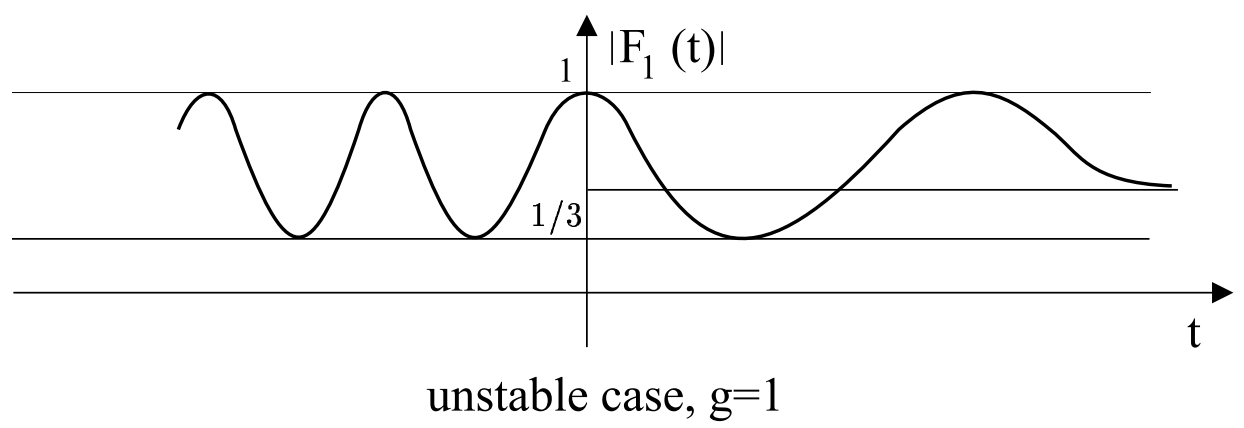




\section{General case}

We assume now that $g$ is an arbitrary real constant. Then $\psi$ no longer has a finite linear decomposition on the Hermite functions, but instead

$$
\psi=\sum_{0}^{\infty} \lambda_{n} \varphi_{n}
$$

with $\sum\left|\lambda_{n}\right|^{2}=1$. Then the following result holds true :

\section{Theorem 3.7}

$$
\left\langle U_{0}(t, 0) \psi_{\beta}, U_{g}(t, 0) \psi_{\beta}\right\rangle=e^{-i(\theta(t)-\theta(0)) \alpha} \sum_{0}^{\infty}\left|\lambda_{n}\right|^{2} e^{-i n(\theta(t)-\theta(0))}
$$

Therefore $|F(t)|=1$ if $\theta(t)-\theta(0)=0(\bmod 2 \pi)$

If $g$ is such that $\alpha:=\frac{1}{2}+\sqrt{\frac{1}{4}+2 g^{2}}=\frac{p}{q} \in \mathbb{Q}$, then $F(t)=1$ if $\theta(t)-\theta(0)=0,(\bmod 2 q \pi)$

\section{Exact Classical Fidelity implies Exact Quantum Fidelity}

Let $x(t)$ and $y(t)$ be real classical solutions for the Hamiltonians $H_{0}(t)$ and $H_{g}(t)$ respectively such that $x(0)=y(0)$ and $\dot{x}(0)=\dot{y}(0)$ which means that the trajectories merge from the same point in phase space at $t=0$

$t$ is said a time of classical fidelity if $x(t)=y(t)$ and $\dot{x}(t)-\dot{y}(t)=0$, and the classical infidelity at time $t$ is measured by the distance $|x(t)-y(t)|$.

Theorem 3.8 Let $x(t)$ be a real solution of Hill's equation $\ddot{x}+f x=0$. We write it as

$$
x(t)=e^{u(t)} \cos \tilde{\theta}(t)
$$

with $u$ and $\tilde{\theta}$ real functions and $\tilde{\theta}(t)=g \sqrt{2} \int_{0}^{t} d s e^{-2 u(s)}$. Then $y(t):=e^{u(t)}$ is a solution of equation

$$
\ddot{y}+f y-\frac{2 g^{2}}{y^{3}}=0
$$

such that $x(0)=y(0)$ and $\dot{x}(0)=\dot{y}(0)$. This means that $y(t)$ is a real trajectory for Hamiltonian $H_{g}(t)$, merging from the same point in phase space as $x(t)$.

We clearly have

$$
|x(t)-y(t)|=y(t)(1-\cos \tilde{\theta}(t))
$$

which vanishes for $\tilde{\theta}(t)=0(\bmod 2 \pi)$ 
Remark 3.9 By choosing $\epsilon$ such that $e^{2 \epsilon}=g \sqrt{2}$, we clearly have

$$
\tilde{\theta}(t)=\theta(t)-\theta(0)
$$

Corollary 3.10 If $\theta(t)-\theta(0)=0(\bmod 2 \pi)$, then the classical fidelity is zero, and the Quantum Fidelity equals 1 (at least in absolute value in the case of general g).

The proof is very elementary. Let $z(t)$ be a complex solution of Hill's equation of the form

$$
z(t):=e^{u(t)+i \tilde{\theta}(t)}
$$

with $u$ and $\tilde{\theta}$ real functions. Since $f$ is real the wronskian of $z$ and $z^{*}$ is constant, and we assume that it equals $4 i \sqrt{g}$ :

$$
\dot{z} z^{*}-\dot{z}^{*} z=2 i \tilde{\theta}|z|^{2}=4 i \sqrt{g}
$$

Therefore

$$
\frac{d}{d t} \tilde{\theta}=g \sqrt{2} e^{-2 u}
$$

and therefore Hill's equation for $z$ implies :

$$
\ddot{z}+f z=0=\left[\ddot{u}+\dot{u}^{2}-\left(\frac{d}{d t} \tilde{\theta}\right)^{2}+i\left(\frac{d^{2}}{d t^{2}} \tilde{\theta}+2 \dot{u} \frac{d}{d t} \tilde{\theta}\right)\right] z
$$

whence the equation for $u$ :

$$
\ddot{u}+\dot{u}^{2}-2 g^{2} e^{-4 u}=-f
$$

and thus for $y:=e^{u}$ :

$$
\ddot{y}+f y-\frac{2 g^{2}}{y^{3}}=0
$$

which is nothing but Newton's equation for Hamiltonian $H_{g}(t)$. Furthermore assuming that $\tilde{\theta}(0)=0, x(t):=e^{u(t)} \cos \tilde{\theta}(t)$ and $y(t)$ have the same initial data. This completes the proof, noting that

$$
\tilde{\theta}(t)=\theta(t)-\theta(0)=g \sqrt{2} \int_{0}^{t} d s e^{-2 u(s)}
$$

\section{Références}

[1] Benenti G., Casati G., Sensitivity of Quantum motion for Classically Chaotic Systems, arXiv : quant-ph/0112060 (2001)

[2] Benenti G., Casati G., Quantum-classical Correspondence in Perturbed Chaotic Systems, Phys. Rev. E, 65 066205-1 (2002) 
[3] Benenti G., Casati G., Veble G, Asymptotic Decay of the Classical Loschmidt Echo in Chaotic Systems, arXiv : nlin.CD/0208003 (2002)

[4] Benenti G., Casati G., Veble G., On the Stability of Classically Chaotic Motion under System's Perturbations, Phys. Rev. E 67055202 - (R) (2003)

[5] Cerruti N., Tomsovic S., Sensitivity of Wave Field Evolution and Manifold Stability in Chaotic Systems, Phys. Rev. Lett. 88054103 (2002)

[6] Cerruti N., Tomsovic S., A Uniform Approximation for the Fidelity in Chaotic Systems, J. Phys. A : Math. Gen.36 3451-3465 (2003)

[7] Combescure M., The Quantum Fidelity for the time-dependent Singular Quantum Oscillator to appear (2004)

[8] Combescure M., Robert D., Semiclassical Spreading of Quantum Wavepackets and Applications near Unstable Fixed Points of the Classical Flow, Asymptotic Analysis, 14 377-404, (1997)

[9] Combescure M., Robert D., A Phase-space Study of Quantum "Fidelity" in the Semiclassical limit in preparation (2004)

[10] Cucchietti F. M., Pastawski H. M., Wisniacki D. A., Decoherence as Decay of the Loschmidt Echo in a Lorentz Gas, Phys. Rev. E, 65045206(R) (2002)

[11] Cucchietti F. M., Pastawski H. M., Jalabert R. A., Universality of the Lyapunov Regime for the Loschmidt Echo, arXiv : cond-mat/0307752 (2003)

[12] Cucchietti F. M., Dalvit D. A., Paz J. P., Zurek W. H., Decoherence and the Loschmidt Echo, arXiv :quant-phys/0306142 (2003)

[13] Eckhardt B., Echoes in Classical Dynamical Systems, J. Phys. A : Math. and general, 36, 371-380 (2003)

[14] Emerson J., Weinstein Y. Lloyd S., Cory D., Fidelity Decay as an Indicator of Quantum Chaos, Phys. Rev. Lett. 89284102 - (2002)

[15] Fiete G. A., Heller E. J., Semiclassical Theory of Coherence and Decoherence, Phys. Rev. A 68022112 (2003)

[16] Giovannetti V., Llyod S., Maccone L., Quantum Limits to Dynamical Evolution, Phys. Rev. A 052109 - (2003) 
[17] Hörmander L., Symplectic Classification of Quadratic Forms and General Mehler Formulas Math. Zeitschrift, (1995)

[18] Jacquod P., Adagideli I., Beenakker C. W., Decay of the Loschmidt Echo for Quantum States with sub-Planck scale Structures, Phys. Rev. Lett. 89154103 (2002)

[19] Jacquod P., Adagideli I., Beenakker C. W., Anomalous Power Law of Quantum Reversibility for Classically Regular Dynamics, Europhys. Lett. 61 729-735 (2003)

[20] Jalabert R. A., Pastawski H. M., Environment-independent decoherence Rate in Classically Chaotic Systems, arXiv : cond-mat/0010094 (2001)

[21] Jalabert R. A., Pastawski H. M., Environment-Independent Decoherence Rate in Classically Chaotic Systems, Phys. Rev. Lett. 86 2490-2493, (2001)

[22] Mehlig B., Wilkinson M., Semiclassical trace formulae using coherent states Ann. Phys. (Leipz)10 541- (2001)

[23] A. Perelomov, Generalized Coherent States and their Applications, SpringerVerlag, (1986)

[24] Peres A., Stability of Quantum motion in Chaotic and Regular Systems Phys. Rev. A 30, 1610-1615 (1984)

[25] Prosen T., On General Relation Between Quantum Ergodicity and Fidelity of Quantum Dynamics, arXiv :quant-ph/0106149 (2001)

[26] Prosen T., Seligman T. H., Decoherence of Spin Echoes, arXiv :nlin.CD/0201038 (2002)

[27] Prosen T., Seligman T. H., Znidaric M., Stability of Quantum Coherence and Correlation Decay, Phys. Rev. A 67042112 - (2003)

[28] Prosen T., Seligman T. H., Znidaric M., Theory of Quantum Loschmidt Echoes, arXiv : quant-ph/0304104, (2003)

[29] Prosen T., Znidaric M., Stability of Quantum Motion and Correlation Decay, J. Phys. A , Math. Gen, 35, 1455-1481 (2002) 
[30] Robinett R. W. Quantum wave packet revivals, Phys. Rep. 392, 1-119, (2004)

[31] Sankaranarayanan R., Lakshminarayan A., Recurrence of Fidelity in NearIntegrable Systems, Phys. Rev. E 68036216 - (2003)

[32] Schlunk S., d'Arcy M. B., Gardiner S. A., Cassettari D., Godun R. M., Summy G. S., Signatures of quantum stability in a classically chaotic system, Phys. Rev. Lett. 90 124102- (2003)

[33] Silvestrov P. G., Tworzydlo J., Beenakker C. W., Hypersensitivity to Perturbations of Quantum-Chaotic Wavepacket Dynamics, Phys. Rev. Lett. 67 025204(R) (2003)

[34] Vanicek J., Cohen D., Survival Probability and Local Density of States for One-dimensional Hamiltonian Systems, J. Phys. A : Math. Gen. 36 9591-9608 (2003)

[35] Vanicek J., Heller E. J., Semiclassical Evaluation of Fidelity in the FermiGolden-Rule and Lyapunov Regimes, Phys. Rev. E 68 056208- (2003)

[36] Vanicek J., Heller E. J., Uniform Semiclassical Wave Function for Coherent $2 D$ Electron Flow, arXiv :nlin.CD/0209001 (2002)

[37] Veble G., Prosen T., Faster than Lyapunov Decays of Classical Loschmidt Echo, Phys. Rev. Lett. .....(2003)

[38] Wang Wen-ge, Baowen Li, Crossover of Quantum Loschmidt Echo from Golden Rule Decay to Perturbation-Independent Decay, Phys. Rev. E 66056208 (2002)

[39] Wang Wen-ge, Casati G., Baowen Li, Stability of Quantum Motion : Beyond Fermi-golden-rule and Lyapunov Decay, arXiv :quant-ph/0309154 (2003)

[40] Weinstein Y., Lloyd S., Tsallis C., The Edge of Quantum Chaos, Phys. Rev. Lett. 89 214101- (2002)

[41] Weinstein Y., Emerson J., Lloyd S., Cory D ?., Fidelity Decay Saturation Level for Initial Eigenstates, arXiv :quant-ph/0210063 (2002)

[42] Weinstein Y., Lloyd S., Tsallis C., Border between Regular and Chaotic Quantum Dynamics, Phys. Rev. Lett. 89 214101-1,4 (2002) 
[43] Wisniacki D., Short time Decay of the Loschmidt Echo, Phys. Rev. E 67 016205- (2003)

[44] Wisniacki D., Cohen D., Quantum Irreversibility, Perturbation Independenbt Decay, ans the parametric Theory of the Local Density of States, Phys. Rev. E 66046209 (2002)

[45] Znidaric M., Prosen T., Fidelity and Purity Decay in Weakly Coupled Composite Systems, J. Phys. A 362463-2481 (2003) 